\title{
START-TO-END JITTER SIMULATIONS OF THE LINAC COHERENT LIGHT SOURCE*
}

\author{
M. Borland ${ }^{\dagger}$, Y.-C. Chae, S. Milton, R. Soliday (Argonne National Laboratory) V. Bharadwaj, \\ P. Emma, P. Krejcik, C. Limborg, H.-D. Nuhn, M. Woodley (Stanford Linear Accelerator Center)
}

\begin{abstract}
The Linac Coherent Light Source (LCLS) is a fourthgeneration light source demonstration project based on the self-amplified spontaneous emission (SASE) free-electron laser (FEL) concept. It will combine a new photoinjector, the Stanford Linear Accelerator Center (SLAC) linac, with two stages of bunch compression and a long undulator to create intense radiation pulses at $1.5 \mathrm{~A}$. Successful operation of the LCLS will require consistent delivery of a highbrightness electron beam to the undulator, in the face of effects such as wakefields and coherent synchrotron radiation (CSR). Because of the sensitivity to beam quality and subtle effects, it is necessary to perform integrated tracking from the cathode through the undulator. We report on the combined use of PARMELA, elegant, and GENESIS to perform these simulations, and in particular the simulation of pulse-to-pulse variation in FEL performance due to rfand laser-related variation in the electron beam.
\end{abstract}

\section{INTRODUCTION}

As was recently demonstrated experimentally [1], it is possible to build a linac-driven SASE FEL that reaches saturation. However, even in this relatively small demonstration, jitter in the electron beam was a significant issue. Jitter will be even more critical in the LCLS [2], which has many more components, several stages of bunch compression, and more stringent requirements on beam quality. For the LCLS, 1-nC bunches must be accelerated to $14.35 \mathrm{GeV}$ while being compressed to an rms length of $22 \mu \mathrm{m}$ (73 fs) with normalized slice emittances maintained below $1.2 \mu \mathrm{m}$ and rms energy spread below $0.02 \%$. Simulation of jitter effects is important to provide tolerance specifications and realistic predictions of performance.

The new 150-MeV LCLS photoinjector (PI) will deliver beam via a dogleg to the existing SLAC linac (L1), which delivers a $250-\mathrm{MeV}$ beam to the $\mathrm{BC} 1$ double-chicane bunch compressor. Here, the $0.83-\mathrm{mm}$ rms bunch length is compressed to $0.19 \mathrm{~mm}$. The $\mathrm{L} 2$ linac accelerates to $4.54 \mathrm{GeV}$, where a double-chicane compressor (BC2) compresses to $22 \mu \mathrm{m}$, after which L3 accelerates to $14.35 \mathrm{GeV}$ for delivery into the undulator hall via a dogleg.

Many of the physical effects at work in the LCLS show a sensitivity to details in the phase space distribution of the

\footnotetext{
* Work supported by U.S. Department of Energy, Office of Basic Energy Sciences, under Contract No. W-31-109-ENG-38 and DE-AC0376SF00515.

† borland@aps.anl.gov
}

beam. For example, CSR effects are very sensitive to the longitudinal distribution. In turn, any emittance growth due to CSR can dramatically affect the FEL. Clearly, then, it is desirable to perform integrated simulations that include effects from the cathode to the undulator, including effects of departure from ideal running conditions. Just as clearly, details of how one code simulates some physical phenomenon might impact the predictions in an unexpected way.

In light of these observations, a collaborative effort was undertaken by ANL, SLAC, and Lawrence Berkeley Laboratory to perform "start-to-end" (S2E) simulations of the LCLS. Our primary goal was a single S2E simulation of the LCLS using one set of codes, a goal that we achieved using the codes PARMELA [3], elegant [4], and GENESIS [5]. A secondary, unrealized goal was to create a suite of interchangeable codes that could be used for these simulations in order to provide cross-checks. A mechanism was demonstrated that would make this goal achievable.

\section{SIMULATION METHODS}

Rather than build a single code for these simulations, we developed a systematic way to transfer the results of one code to another for continuation of the simulations. This method is based on the use of the ANL-developed SelfDescribing Data Sets (SDDS) file protocol [6], which was previously used for multi-code simulation.

Use of SDDS files has many advantages. They provide a fixed interface between codes while allowing codes to change, provided that authors do not change the names of quantities in the files. The SDDS Toolkit, a software suite that works with SDDS files, allows transformation of SDDS files to provide translation between coordinate systems used by different codes. The suite is commandlinebased and can be used in scripts, allowing a high degree of automation. The Toolkit also provides common postprocessing tools, so that even users of multiple codes may use a single set of postprocessing tools. Finally, SDDS binary files after version 1.19 are cross-platform readable, and the Toolkit runs on UNIX and Windows operating systems.

PARMELA was used for the PI because it is an accepted code for such applications. Unfortunately, PARMELA is not SDDS-compliant and apparently cannot be made so. It is not available for Unix workstations, limiting our ability to include the PI fully in the jitter simulations. We performed a single PARMELA simulation for nominal conditions, manually translated the data into SDDS, and used these data as input to the rest of the process. PI jitter was emulated by randomizing the arrival time, charge, position, 
and energy of the PARMELA-generated distribution.

The 6-D tracking code elegant was used for the remainder of the linac, starting at an energy of $150 \mathrm{MeV}$, where space charge forces should be negligible. elegant is SDDS-compliant and hence easy to use in an automated, concurrent fashion on a multiworkstation queue, which is necessary given the scope of the simulations. elegant includes many physical effects that are important in the LCLS, including: rf elements with exact time and energy dependence, as well as end focusing; transverse and longitudinal wakefields of accelerating structures; resistive wall wakefields; coherent and incoherent synchrotron radiation effects in dipoles; CSR effects in drift spaces following dipoles; and residual dispersion correction. elegant supports perturbation of the accelerator, including the ability to perturb the rf structure phases and voltages consistent with the number and arrangement of the klystrons. This is accomplished using a script that creates SDDS files, giving sets of phases and voltages for the structures.

The code GENESIS was used for FEL simulation. It can operate in either steady state or SASE mode. In SASE mode, a photon slice slips over electron beam slices as the beam traverses an undulator period. Full SASE simulation, including this slippage effect for the LCLS FEL, requires millions of electron beam slices. To reduce run times, we instead used steady-state mode as follows: using the BEAMFILE input to GENESIS, we provided parameters characterizing a series of 20 beam slices, including emittance, centroid position, energy, and energy spread. For each of the slices, GENESIS creates a beam in 6-D phase space for tracking through the FEL and computes the FEL output independently, which is valid for LCLS because each slice is many slippage lengths long.

We modified GENESIS for this work so that it reads the BEAMFILE in SDDS-format [12] and so that it delivers its output in SDDS files. Prior to analysis, any particles with energy deviation of more than $0.3 \%$ from the mean were removed to avoid skewing the averages with low-population tails. (In the vast majority of simulations, no particles are removed. Even with CSR and jitter included, $96 \%$ of cases have less than a $5 \%$ loss of particles.)

\section{SIMULATION RESULTS}

Having created the mechanism for interfacing these codes, we executed 150 randomized simulations with elegant using the beam from PARMELA, which consisted of 100-k macroparticles. Errors were generated from 2-sigma gaussian distributions using the rms levels given in Table 1. SDDS-based scripts were written to automate the preparation of these runs and submit them to a distributed queue of 60 Sun workstations. Additional SDDS-based scripts performed the slice analysis to prepare the BEAMFILEs for GENESIS, after which GENESIS runs were submitted to the same queue. Analysis, display, and merging of elegant and GENESIS output were performed using SDDS-based scripts. Having all output in SDDS files auto-
Table 1: RMS Error Levels for Jitter Simulations. Initial quantities refer to the exit of the PI, at $150 \mathrm{MeV}$.

\begin{tabular}{|l|l|}
\hline Quantity & RMS Error Level \\
\hline \hline initial $\mathrm{x}, \mathrm{y}$ centroid & $2.1 \mu \mathrm{m}(1 \%$ of size $)$ \\
\hline initial $\mathrm{x}^{\prime}, \mathrm{y}^{\prime}$ centroid & $0.19 \mu \mathrm{r}(1 \%$ of divergence $)$ \\
\hline initial t centroid & $0.9 \mathrm{ps}$ \\
\hline initial energy & $0.1 \%+$ timing/phase contrib. \\
\hline charge & $2 \%$ \\
\hline L1 phase, voltage & 0.1 deg-S, $0.1 \%$ \\
\hline L2, L3 phase & 0.07 deg-S \\
\hline L2, L3 voltage & $0.07 \%, 0.05 \%$ \\
\hline $\mathrm{x}$-band phase, voltage & 0.3 deg-X, $0.25 \%$ \\
\hline BC1 dipoles & $0.02 \%$ (ganged) \\
\hline BC2 dipoles & $0.05 \%$ (ganged) \\
\hline DL dipoles & $0.01 \%$ (ganged) \\
\hline
\end{tabular}

mated the analysis of correlations, presented below.

Two sets of runs are reported here: one with CSR and one without. The former shows severe emittance growth, caused by a CSR-driven microbunch instability that corrupts the slice emittances. We note that the CSR model [7] [8] used by elegant may not be fully applicable to the LCLS parameters. Although there is experimental evidence that this model works [9] [10], the LCLS is in a different regime than these experiments. In particular, downstream of the second bunch compressor, where most of the CSR-induced emittance growth occurs, the bunch length is very short $(22 \mu \mathrm{m})$ compared to the transverse beam size $(\sim 1 \mathrm{~mm})$. Hence, the 1-D line-charge model may not apply to all of the LCLS [11], and by using it, we may overestimate the emittance growth. The limitations of this model may be very significant and are not yet fully understood.

Table 2 shows simulation results for four cases: two reference cases without jitter and two cases with jitter. Most results are from elegant, but the gain length and output power from GENESIS are also shown. With the exception of the GENESIS results, all data are averaged over the "core slices" of the beam, which are the central 16 of the 20 slices. Because of the frequently highly skewed nature of the distributions, we quote median values and quartile half-ranges rather than means and standard deviations.

The jitter results without CSR show that acceptable performance is possible given the assumed jitter levels, which, while challenging, are not unreasonable. Because of the large number of randomizations, it is possible to perform correlation analysis to determine the cause of variation in, say, the gain length computed by GENESIS. This shows that $80 \%$ of the variation in gain length results from jitter in the timing of the beam from the PI. This finding is demonstrated in Fig. 1, where we show a scatter plot of the timing error and the gain length, along with other data. Also shown are the core-slice-averaged horizontal emittance, current, and energy spread. The variation in gain 
Table 2: Core-slice-averaged median values and quartile half-ranges for nominal and jitter results from elegant, and slice-averaged FEL results from GENESIS (last two columns). $\Delta t_{80}$ is the total length of the core slices, $\sigma_{\delta}$ is the rms energy spread, $\epsilon_{n, x}$ is the normalized rms horizontal emittance, $\lambda$ is the light wavelength, $L_{g}$ is the gain length, and $P_{\text {out }}$ is the output radiation power.

\begin{tabular}{|c|c|c|c|c|c|c|c|c|}
\hline jitter & CSR & Current & $\Delta t_{80}$ & $\sigma_{\delta}$ & $\epsilon_{n, x}$ & $\lambda$ & $L_{g}$ & $P_{\text {out }}$ \\
\hline$?$ & $?$ & $\mathrm{kA}$ & $\mathrm{ps}$ & $10^{-4}$ & $\mu m$ & $\mathrm{~A}$ & $\mathrm{~m}$ & $\mathrm{GW}$ \\
\hline \hline no & no & 3.9 & 0.20 & 0.9 & 0.68 & 1.500 & 3.1 & 12.2 \\
\hline yes & no & $3.8 \pm 0.6$ & $0.21 \pm 0.04$ & $0.9 \pm 0.3$ & $0.68 \pm 0.01$ & $1.499 \pm 0.003$ & $3.2 \pm 0.2$ & $11.4 \pm 2.6$ \\
\hline no & yes & 4.0 & 0.20 & 3.0 & 3.13 & 1.502 & 9.7 & 0.7 \\
\hline yes & yes & $4.3 \pm 1.0$ & $0.19 \pm 0.05$ & $3.1 \pm 1.0$ & $3.16 \pm 0.50$ & $1.502 \pm 0.004$ & $9.5 \pm 0.8$ & $1.1 \pm 0.4$ \\
\hline
\end{tabular}

length, while caused fundamentally by the variation in arrival time from the PI, is related to variation in current and is not strongly affected by variations in emittance and energy spread, which counter the effect of current variations.

We noted earlier that we were unable to extend the jitter simulations into the photoinjector because of limitations of PARMELA. Our results make it clear that it is important to make this extension. Indeed, one should include a model of the laser itself, as pulse-to-pulse temporal and spatial variation in the laser may be important.

\section{CONCLUSION}

We have developed a powerful mechanism for exploring the behavior of a complex accelerator and FEL. Multiple codes are used, each in its regime of applicability. SDDS files facilitate interfacing between codes and analysis of results, providing a fixed interface between codes that may themselves change. With the jitter results, SDDS tools can be used to easily explore correlations among accelerator inputs and FEL outputs, giving insights that will help focus efforts to optimize the design. Our results show that it is important to include the photoinjector fully in the jitter simulations.
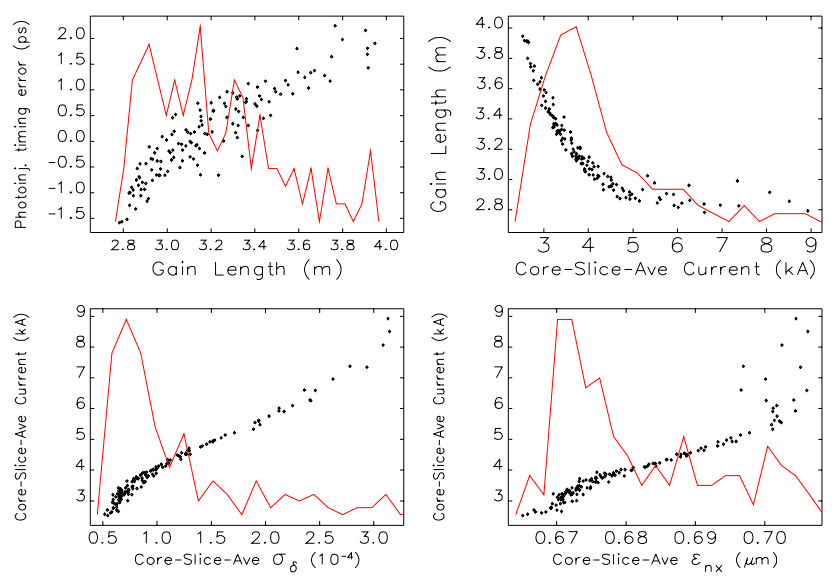

Figure 1: Results of jitter simulations without CSR. Lines show histograms of the quantities along the horizontal axis.
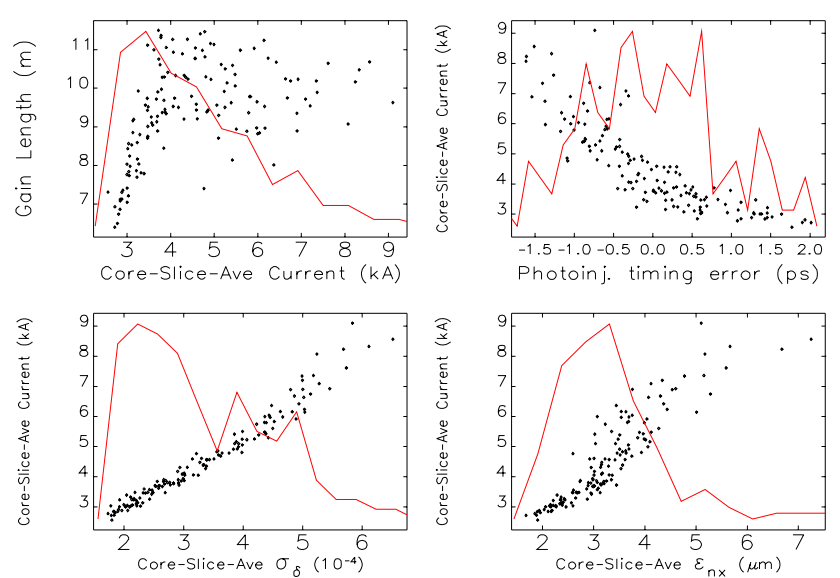

Figure 2: Results of jitter simulations with CSR. Lines show histograms of the quantities along the horizontal axis.

\section{REFERENCES}

[1] S. V. Milton, et al., Science 10.1126/science.1059955, Published Online (May 17, 2001).

[2] LCLS Design Study Report, SLAC-R-521 (1998).

[3] J. Billen, PARMELA, Los Alamos National Laboratory report LA-UR-96-1835 (1996).

[4] M. Borland, APS LS-287, September 2000. http://www.aps.anl.gov/techpub/lsnotes/lsnotesTOC.html

[5] S. Reiche, NIM A429 (1999) 242.

[6] M. Borland, Proc. 1995 PAC, pp. 2184-2186 (1996).

[7] E. L. Saldin, et al., NIM A 398, 373 (1997).

[8] M. Borland, "Simple Method for Particle Tracking with Coherent Synchrotron Radiation," PRST-AB, to be published.

[9] M. Borland, et al., "Initial Characterization of Coherent Synchrotron Radiation Effects in the Advanced Photon Source Bunch Compressor," Proc. 2001 PAC.

[10] H. H. Braun, et al., "Recent Experiments on the Effect of Coherent Synchrotron Radiation on the Electron Beam of CTF II,” Proc. 2001 PAC.

[11] Derbenev, et al., "Microbunch Radiative Tail-Head Interaction," TESLA-FEL-95-05, Eq. (11).

[12] Y. Chae, et. al, "Development of SDDS-Compliant GENESIS and Its Application,” Proc. 2001 PAC. 